\title{
Model of tunnelling through double quantum layer in a magnetic field
}

\author{
D. L. Meynster, I. Y. Popov, A. I. Popov \\ ITMO University, Kronverkskiy, 49, St. Petersburg, 197101, Russia \\ mdldml@gmail.com,popov1955@gmail.com,popov239@gmail.com
}

PACS 73.21.Ac; 02.30.Tb

DOI 10.17586/2220-8054-2017-8-2-194-201

Several explicitly solvable models are constructed for electron tunneling in a system of double two-dimensional periodic arrays of quantum dots with two laterally coupled leads in a homogeneous magnetic field are constructed. The theory of self-adjoint extensions of symmetric operators is used for modelling of electron transport. Dependencies of the transmission coefficient on the magnetic field, the energy of electron and the distance between layers are investigated. The results are compared with those of tunnelling through the corresponding single-layer periodic arrays.

Keywords: quantum dots array, magnetic field, scattering.

Received: 16 February 2017

Revised: 18 March 2017

\section{Introduction}

Since the publication of famous Hofstadter paper [1], research interest in the spectral properties of twodimensional periodic arrays in a magnetic field has greatly increased. The fractal structure of the spectrum has been a theoretical result for long time, but subsequently, experimental confirmation was obtained. Electron tunneling through periodic array of quantum dots in a homogeneous magnetic field has been intensively investigated over the last few years because it can be relevant for nanotechnology applications [2-4].

There are several different approaches for building models of quantum dot arrays. In this paper, the zero-range potential model $[5,6]$ based on the theory of self-adjoint extensions of symmetric operators is used (see, e.g., [7-12] and references in [13]). One of its benefits is that the model is explicitly solvable.

In double-layer lattices, the layer number provides an additional degree of freedom, which leads to some interesting experimentally measurable physical effects [14]. We suggest several explicitly solvable models for double two-dimensional layer of quantum dots using the approach described in [15].

Because of high quality of nanostructure devices, large Fermi wave length (i.e. the de Broglie wavelength of electrons with energy close to the Fermi energy) $\left(4 \times 10^{-8} \mathrm{~m}\right)$ and long mean free path of electron $\left(10^{-5} \mathrm{~m}\right)$ can be observed. Therefore, one deals with the ballistic regime of electron transport. In this case, the Landauer-Buttiker formalism can be used to derive the conductivity $\sigma$ for the nanostructure with several leads from the transmission coefficient $\left.T(E)\right|_{E=E_{F}}$ (here $E_{F}$ is the Fermi energy).

In the simplest case (one incoming and one outgoing channel), the Landauer formula has the form $\sigma=\frac{e^{2} T}{\hbar(1-T)}[16]$. Here, $e$ is the electron charge, $\hbar$ is the Planck's constant. The geometry of a nanostructure can influence transmission coefficient significantly [17].

In this article we, consider tunneling in the system consisting of double two-dimensional periodic array (with square or hexagonal (honeycomb) lattices in each layer) of quantum dots with two connected semi-infinite leads orthogonal to the plane of the array. We study the influence of the magnetic field and tunneling electron energy on the transmission coefficient and compare our results with the tunneling through the corresponding singlelayer periodic arrays studied earlier $[18,19]$. The main ideas of the present paper was described in an extended abstract [20].

We start from the Hamiltonian of a single particle in constant homogeneous magnetic field $B$. Let us assume that particle has mass $m$ and electric charge $e$. Since the space is $\mathbb{R}^{3}$, we choose a standard basis $\boldsymbol{i}, \boldsymbol{j}, \boldsymbol{k}$ such that $\boldsymbol{B}$ is collinear to $\boldsymbol{k}: \boldsymbol{B}=B \boldsymbol{k}, B \neq 0$. Then, the particle can move freely along $z$ axis, and the state space of the model is $L^{2}\left(\mathbb{R}^{2}\right)$, where $\mathbb{R}^{2}$ is the plane based on vectors $\boldsymbol{i}, \boldsymbol{j}$.

The Hamiltonian $H_{0}$ has the following form:

$$
H_{0}=\frac{1}{2 m}\left(\hat{p}-\frac{e}{c} \boldsymbol{A}(\boldsymbol{r})\right),
$$

where $c$ is the speed of light, $\hat{p}=-i \hbar \nabla$ is the two-dimensional momentum operator, $\boldsymbol{A}(\boldsymbol{r})$ is a vector potential of the field $\boldsymbol{B}(\boldsymbol{B}=\operatorname{rot} \boldsymbol{A})$. Vector potential is not unique, we will use the symmetric gauge $\left(\boldsymbol{A}(\boldsymbol{r})=\frac{1}{2} \boldsymbol{B} \times \boldsymbol{r}\right)$. 
The following standard notations are used: $\omega=|e B| / \mathrm{cm}$ is the cyclotron frequency; $\Phi_{0}=2 \pi \hbar c /|e|$ is the quantum of the magnetic flux; $\xi= \pm B / \Phi_{0}-$ number of the flux quanta through the unit area in $\mathbb{R}^{2}$. The sign of $\xi$ is chosen in such a way that the condition $\xi e B>0$ holds. We will also use the system of units in which $e=\hbar=m=c=1$, to simplify the calculations. Then, $H_{0}$ can be rewritten in the following form:

$$
H_{0}=-\frac{1}{2}\left[\left(\frac{\partial}{\partial x}+\pi i \xi y\right)^{2}+\left(\frac{\partial}{\partial y}-\pi i \xi x\right)^{2}\right]
$$

We will also need the Green function of $H_{0}$, which has the form ( [6]):

$$
G_{0}\left(\boldsymbol{r}, \boldsymbol{r}^{\prime}, E\right)=\frac{1}{2 \pi} \Gamma\left(\frac{1}{2}-\frac{E}{\omega}\right) \exp \left(-\pi i \boldsymbol{r} \wedge \boldsymbol{r}^{\prime}-\frac{1}{2} \pi \xi\left\|\boldsymbol{r}-\boldsymbol{r}^{\prime}\right\|^{2}\right) \times \Phi\left(\frac{1}{2}-\frac{E}{\omega}, 1, \pi \xi\left\|\boldsymbol{r}-\boldsymbol{r}^{\prime}\right\|^{2}\right) .
$$

Here, $\Gamma(x)$ is the Euler Gamma-function, $\Phi(a, c, x)$ is the confluent hypergeometric function of the second kind [21].

\section{Tunnelling through double layer}

Note that different explicitly solvable models can be built here, depending on how electron tunneling between layers is considered (see Fig. 1 and Fig. 2). Both model types will be built here for hexagonal and square lattices.

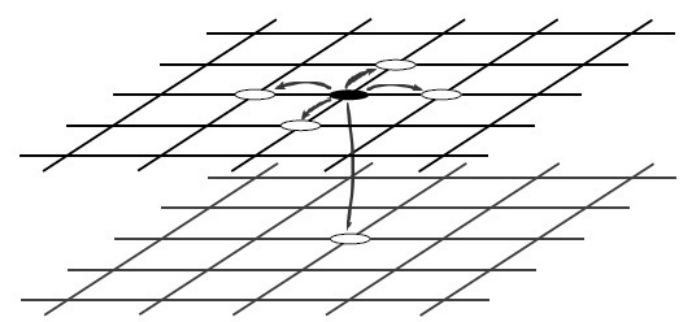

FIG. 1. Model with immediate tunnelling between layers

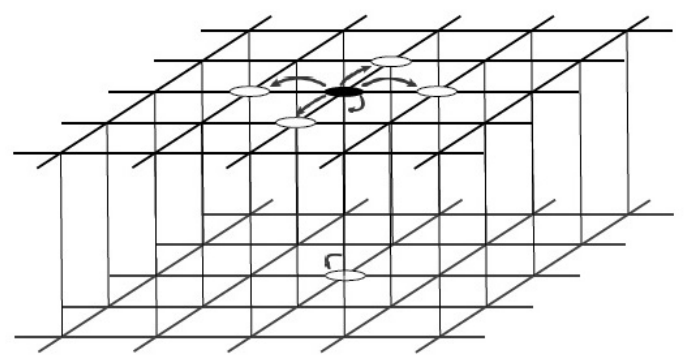

FIG. 2. Model with channel between layers represented by wire of finite length

\subsection{Model with immediate tunneling between layers}

First, we build the model for the square lattice. We assume that the state space of the model is a direct sum of two identical state spaces, one for each layer:

$$
\mathcal{H}=L_{2}\left(\mathbb{R}^{2}\right) \oplus L_{2}\left(\mathbb{R}^{2}\right)
$$

Then, the unperturbed Hamiltonian has the following form:

$$
H_{0}=H_{L} \oplus H_{L}
$$

where $H_{L}$ is the Hamiltonian for the single layer with lattice $\Xi$ (see, e.g., [20]).

To use the Krein's resolvent formula, we first restrict operator $H_{L}$ onto the set of smooth functions vanishing at the points of the lattice, let the restricted operator be $S_{L}$. Then, the restriction of $H_{0}$ has the form:

$$
S_{0}=S_{L} \oplus S_{L} \text {. }
$$

The deficiency space for $S_{0}$ is the direct sum:

$$
\mathcal{G}=\mathcal{G}_{L} \oplus \mathcal{G}_{L}
$$

where $\mathcal{G}_{L}=l_{2}(\Xi)$ is the deficiency space for operator $S_{L}$.

In its turn, the Krein Q-function is the direct sum:

$$
Q=Q_{L} \oplus Q_{L},
$$

where $Q_{L}$ is the Krein Q-function for the corresponding single layer.

Let $f$ be a function from $l_{2}(\Xi)$, then the Krein $\Gamma$-function of pair $\left(H_{L}, S_{L}\right)$ for a single layer has the following form:

$$
\left(\Gamma_{L}(\zeta) f\right)(\boldsymbol{r})=\sum_{\gamma \in \Xi} G_{0}(\boldsymbol{r}, \gamma ; \zeta) f(\gamma)
$$


and the Krein $\Gamma$-function for the whole system can be defined as the direct sum:

$$
\Gamma=\Gamma_{L} \oplus \Gamma_{L}
$$

Finally, using the Krein resolvent formula for infinite deficiency indices, we obtain an expression for the Green function of $H$ :

$$
G\left(\boldsymbol{r}, \boldsymbol{r}^{\prime} ; \zeta\right)=G_{0}\left(\boldsymbol{r}, \boldsymbol{r}^{\prime}, \zeta\right)-\sum_{\boldsymbol{\gamma}, \boldsymbol{\gamma}^{\prime} \in \Xi \oplus \Xi}[Q(z)+A]_{\boldsymbol{\gamma}, \boldsymbol{\gamma}^{\prime}}^{-1} G_{0}(\boldsymbol{r}, \boldsymbol{\gamma} ; \zeta) G_{0}\left(\boldsymbol{\gamma}^{\prime}, \boldsymbol{r}^{\prime} ; \zeta\right)
$$

Now, we need a self-adjoint operator. It is related with the properties of $A$. We assume that the probability of tunneling between non-adjacent points of the lattice is negligible. Due to the periodicity of the system, the Hamiltonian of a single layer $H_{L}$ should be invariant with respect to the magnetic translation group transformations (see, e.g., [25]), therefore, we have the following property for the terms of operator $A_{L}$ of the single layer:

$$
A_{L_{\boldsymbol{\lambda}-\boldsymbol{\gamma}, \boldsymbol{\mu}-\boldsymbol{\gamma}}}=\exp (i \pi \xi(\boldsymbol{\gamma} \wedge(\boldsymbol{\lambda}-\boldsymbol{\mu}))) A_{L_{\boldsymbol{\lambda}, \boldsymbol{\mu}}}
$$

As a consequence, it is sufficient to define elements $A_{L_{\lambda, 0}}$ (see [22] for more detailed explanation):

$$
A_{L_{\boldsymbol{\lambda}, 0}}=\alpha\left(\delta\left(\lambda_{1}, 0\right)\left(\delta\left(1, \lambda_{2}\right)+\delta\left(-1, \lambda_{2}\right)\right)+\delta\left(0, \lambda_{2}\right)\left(\delta\left(\lambda_{2}, 1\right)+\delta\left(\lambda_{2},-1\right)\right)\right) .
$$

Here $\alpha$ is some constant which characterizes the intensity of the interaction.

Now, we need to take into account tunneling between layers, hence, operator $A$ takes the following form:

$$
A=\left[\begin{array}{cc}
A_{L} & \alpha I \\
\alpha I & A_{L}
\end{array}\right]
$$

In the case of hexagonal lattice the main change is different magnetic translations group which lead to changes of the form of operator $A_{L}[26]$ :

$$
\begin{aligned}
A_{L_{\boldsymbol{\lambda}_{\boldsymbol{i}} \boldsymbol{\lambda}_{j}}}=\alpha\left[\delta\left(\boldsymbol{\lambda}_{\boldsymbol{i}}-\boldsymbol{a}_{\mathbf{1}}, \boldsymbol{\lambda}_{\boldsymbol{j}}-\boldsymbol{b}\right)+\delta\left(\boldsymbol{\lambda}_{\boldsymbol{i}}-\boldsymbol{a}_{\mathbf{2}}, \boldsymbol{\lambda}_{\boldsymbol{j}}-\boldsymbol{b}\right)+\delta\left(\boldsymbol{\lambda}_{\boldsymbol{i}}-\boldsymbol{a}_{\mathbf{1}}-\boldsymbol{a}_{\mathbf{2}}, \boldsymbol{\lambda}_{\boldsymbol{j}}-\boldsymbol{b}\right)\right. \\
+\exp \left(\frac{2}{3} i \pi \xi\left(\delta\left(\boldsymbol{\lambda}_{\boldsymbol{i}}+\boldsymbol{a}_{\mathbf{1}}-\boldsymbol{b}, \boldsymbol{\lambda}_{\boldsymbol{j}}\right)-\delta\left(\boldsymbol{\lambda}_{\boldsymbol{i}}+\boldsymbol{a}_{\mathbf{2}}-\boldsymbol{b}, \boldsymbol{\lambda}_{\boldsymbol{j}}\right)\right)\right) \\
\left.\quad \times\left(\delta\left(\boldsymbol{\lambda}_{\boldsymbol{i}}+\boldsymbol{a}_{\mathbf{1}}-\boldsymbol{b}, \boldsymbol{\lambda}_{\boldsymbol{j}}\right)+\delta\left(\boldsymbol{\lambda}_{\boldsymbol{i}}+\boldsymbol{a}_{\mathbf{2}}-\boldsymbol{b}, \boldsymbol{\lambda}_{\boldsymbol{j}}\right)+\delta\left(\boldsymbol{\lambda}_{\boldsymbol{i}}+\boldsymbol{a}_{\mathbf{1}}+\boldsymbol{a}_{\mathbf{2}}-\boldsymbol{b}, \boldsymbol{\lambda}_{\boldsymbol{j}}\right)\right)\right] .
\end{aligned}
$$

\subsection{Model with channel between layers}

First, we need to define the state space of this model. As in the previous case, the state space of a single layer is $\mathcal{H}_{L}$. We assume that the distance between layers is $d$. Let $\mathcal{H}_{v}$ be the state space of a single vertical wire, $\mathcal{H}_{v}=L_{2}([0 ; d])$. Obviously, the state space of the whole set of wires is defined as a direct sum:

$$
\mathcal{H}_{V}=\bigoplus_{\gamma \in \Xi} \mathcal{H}_{v}
$$

Then, the whole model has the following state space:

$$
\mathcal{H}=\mathcal{H}_{L} \oplus \mathcal{H}_{V} \oplus \mathcal{H}_{L}
$$

Therefore, the Hamiltonian of the system (if the interaction is switched off) has the form:

$$
H=H_{L} \oplus H_{V} \oplus H_{L}
$$

where $H_{V}=\bigoplus_{\gamma} H_{v_{\gamma}}, H_{v}=-\frac{\partial}{\partial x^{2}}-$ operator under Neumann conditions at points 0 and $d$.

Restrict $H_{v}$ onto $D=\{\varphi \mid \varphi(0)=\varphi(d)=0\}$, if $S_{v}$ is the restricted (symmetric) operator and $S_{V}=\bigoplus_{\gamma} S_{v_{\gamma}}$, then, the Hamiltonian $S=S_{L} \oplus S_{V} \oplus S_{L}$ is the restriction of $H$.

The deficiency space for operator $S_{v}$ is $\mathcal{G}_{v}=\mathbb{C}^{2}$, for $G_{V}: \mathcal{G}_{\mathcal{V}}=\bigoplus_{\gamma} G_{v \gamma}$. Then, for $S$, one has:

$$
\mathcal{G}=\mathcal{G}_{\mathcal{L}} \oplus \mathcal{G}_{\mathcal{V}} \oplus \mathcal{G}_{\mathcal{L}}
$$

The Green function for $H_{v}$ is well-known:

$$
G_{v}\left(x, x^{\prime} ; \zeta\right)=-\frac{1}{2 k \sin (k d)}\left(\cos \left(k\left(d-\left|x^{\prime}-x\right|\right)\right)+\cos \left(k\left(d-\left(x^{\prime}+x\right)\right)\right)\right) .
$$


The Krein $\Gamma$-function for a single wire has the following form:

$$
\left(\Gamma_{v}(\zeta)\left(\begin{array}{l}
\xi_{1} \\
\xi_{2}
\end{array}\right)\right)(x)=\left(\begin{array}{l}
G_{v}(x, 0 ; \zeta) \xi_{1} \\
G_{v}(x, d ; \zeta) \xi_{2}
\end{array}\right) .
$$

Then, $\Gamma_{V}=\bigoplus_{\gamma} \Gamma_{v}$ and $\Gamma=\Gamma_{L} \oplus \Gamma_{V} \oplus \Gamma_{L}$. The Krein $Q$-function for a single wire has the form of $2 \times 2$-matrix:

$$
Q_{v}(\zeta)=\left[\begin{array}{ll}
G_{v}(0,0 ; \zeta) & G_{v}(0, d ; \zeta) \\
G_{v}(d, 0 ; \zeta) & G_{v}(d, d ; \zeta)
\end{array}\right]
$$

Hence, the Krein $Q$-function for the set of wires is $Q_{V}(\zeta)=\bigoplus_{\gamma \in \Xi} Q_{v}(\zeta)$, and for the whole system, we have:

$$
Q(\zeta)=Q_{L}(\zeta) \oplus Q_{V}(\zeta) \oplus Q_{L}(\zeta)
$$

To obtain the matrix of interactions $A$, let us first define matrices $A_{1 V}$ and $A_{2 V}$, which characterize the tunneling from the first or the second layer, correspondingly, to the layer of vertical wires. For the single wire, we have $A_{1 v}=\left[\begin{array}{ll}\alpha & 0\end{array}\right]$. Then, one has $A_{1 V}=\bigoplus_{\gamma \in \Xi} A_{1 v_{\gamma}}$. Similarly, $A_{v 2}=\left[\begin{array}{c}\alpha \\ 0\end{array}\right]$ and $A_{V 2}=\bigoplus_{\gamma \in \Xi} A_{v 2}$. Consider also $A_{V 1}=A_{1 V}^{T}, A_{2 V}=A_{V 2}^{T}$. Finally, we get the following matrix $A$ :

$$
A=\left[\begin{array}{ccc}
A_{L} & A_{1 V} & 0 \\
A_{V 1} & 0 & A_{V 2} \\
0 & A_{2 V} & A_{L}
\end{array}\right]
$$

\section{Model of tunnelling}

Now, we would like to construct the general model of tunneling through the quantum layer (single or double, it doesn't matter). To do that, we use the idea from [27,28]: let $D$ be any device that is connected to a pair of semi-infinite leads at points $C_{-}$and $C_{+}$(Fig. 3) We assume that the Green function $G_{D}$ for $D$ is already known. Let $\mathcal{H}_{D}$ be the state space for the device, $\mathcal{H}_{ \pm}$be the state spaces for leads, then, the state space for the whole system is given as follows:

$$
\mathcal{H}_{0}=\mathcal{H}_{-} \oplus \mathcal{H}_{D} \oplus \mathcal{H}_{+} \cdot
$$

The Hamiltonian of the system without interaction between the device and the channels is just the orthogonal sum of the corresponding operators:

$$
H_{0}=H_{-} \oplus H_{D} \oplus H_{+},
$$

where $H_{D}$ is the Hamiltonian of a charged particle in $D, H_{ \pm}$is the Hamiltonian of the charged particle in space $L^{2}\left(R_{ \pm}\right)$under Neumann conditions at the edge (it has the form $\left.-\frac{\partial}{\partial x^{2}}\right)$. It is easy to find the Green functions for $H_{ \pm}$:

where $k^{2}=\zeta$

$$
G_{ \pm}\left(x, x^{\prime} ; \zeta\right)=\frac{i}{2 k}\left[\exp \left(i k\left|x-x^{\prime}\right|\right)+\exp \left( \pm i k\left(x+x^{\prime}\right)\right)\right]
$$

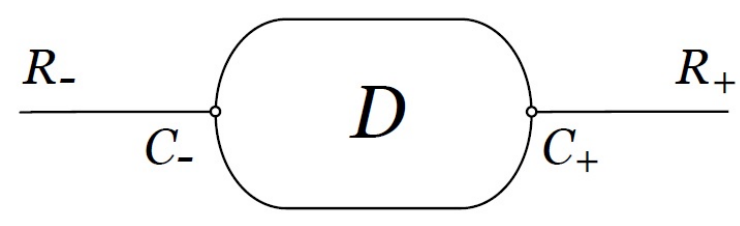

FIG. 3. Model of the device with connected leads

Using the theory of self-adjoint extensions again, we can obtain the model Hamiltonian $\mathcal{H}$. To do that, we, first, restrict $H_{0}$ onto the set of smooth functions vanishing at the points $C_{ \pm}$, then, build its extension $H$.

After solving the scattering problem for $H$, we obtain the following formula for the transmission coefficient $T(E)$ :

$$
T(E)=\frac{\left|\left(\alpha_{-} \alpha_{+} Q_{21}^{D}\right)^{2}\right|}{E|\operatorname{det}[Q(E)+A]|^{2}}
$$


Here $A$ is the matrix of the self-adjoint operator that characterizes the extension,

$$
A=\left[\begin{array}{cccc}
0 & \alpha_{-} & 0 & 0 \\
\alpha_{-} & 0 & 0 & 0 \\
0 & 0 & 0 & \alpha_{+} \\
0 & 0 & \alpha_{+} & 0
\end{array}\right]
$$

$Q(E)$ is the Krein Q-function:

$$
Q(E)=\left[\begin{array}{cccc}
Q_{-}(E) & 0 & 0 & 0 \\
0 & Q_{D}^{11}(E) & Q_{D}^{12}(E) & 0 \\
0 & Q_{D}^{21}(E) & Q_{D}^{22}(E) & 0 \\
0 & 0 & 0 & Q_{+}(E)
\end{array}\right]
$$

$Q_{ \pm}(E)=G_{ \pm}(0,0 ; E)$ and $Q_{D}$ is $2 \times 2$ Krein Q-function for the extension, where $Q_{D}^{12}(E)=\overline{Q_{D}^{21}(E)}=$ $G_{D}\left(\boldsymbol{r}_{\mathbf{1}}, \boldsymbol{r}_{\mathbf{2}} ; E\right)$ and $Q_{D}^{11}(E), Q_{D}^{22}(E)$ are the regular parts of $G_{D}\left(r_{1}, r_{1} ; E\right), G_{D}\left(r_{2}, r_{2} ; E\right)$, correspondingly. Values $\alpha_{ \pm}$describe the quality of contacts $C_{ \pm}$. This technique was used in [18] to construct a model of tunneling through single quantum electron layer.

\section{Results and comparison with single-layer arrays}

For numerical experiments in this section, constants $\alpha, \alpha_{-}, \alpha_{+}$were all selected to be equal to 1 . Only a finite fragment of an infinite lattice is used for calculations since points which are far enough from contacts don't have much influence on transmission coefficient value. For the square lattice, the basic vectors $a_{1}=(1,0), a_{2}=(0,1)$ were used; the hexagonal (honeycomb) lattice is presented as a sum of two square lattices.

The transmission coefficient $T$ is calculated as a function of electron energy $E$ for different values of the magnetic field $B$. The resulting function of two values is represented as a contour plot. We assume that both contacts are in the same lattice cell, calculations show that putting them in different cells of the lattice leads to decreasing of $T$ but doesn't add or remove any other significant effects.

During the investigation of tunneling through single-layer lattices, wide zones in the resulting contour plot with very low value of $T$ were discovered $[18,19]$. The widths of these zones are greater than the product of the Boltzmann constant and the temperature value and, thus, this phenomenon is physically measurable. Our calculations show that these zones are preserved both in the square and the hexagonal lattice cases (see Figs. 4, 5, 6 and 7). Plots were built for the model with direct tunneling between the layers. The model with channels between the layers gives one qualitatively analogous result - such zones exist too.

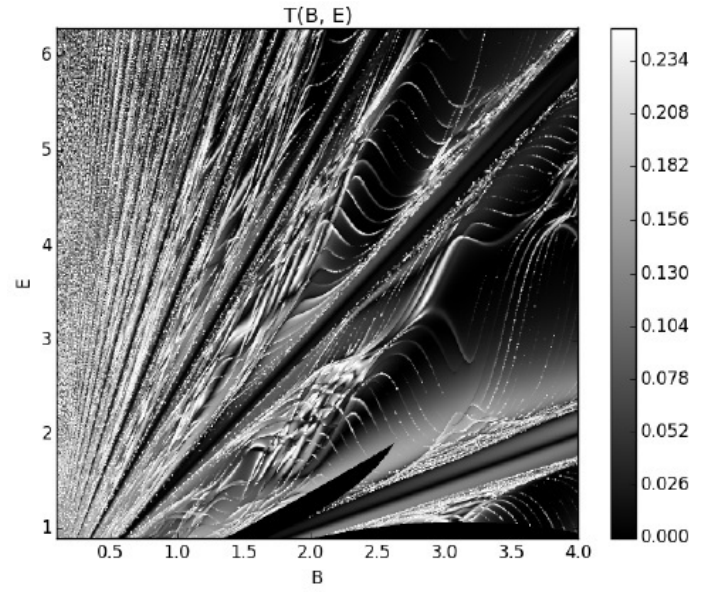

FIG. 4. Dependence of transmission coefficient value $T$ on electron energy $E$ and magnetic field $B$ : case of single-layer square lattice. $B$ and $E$ are in dimensionless units

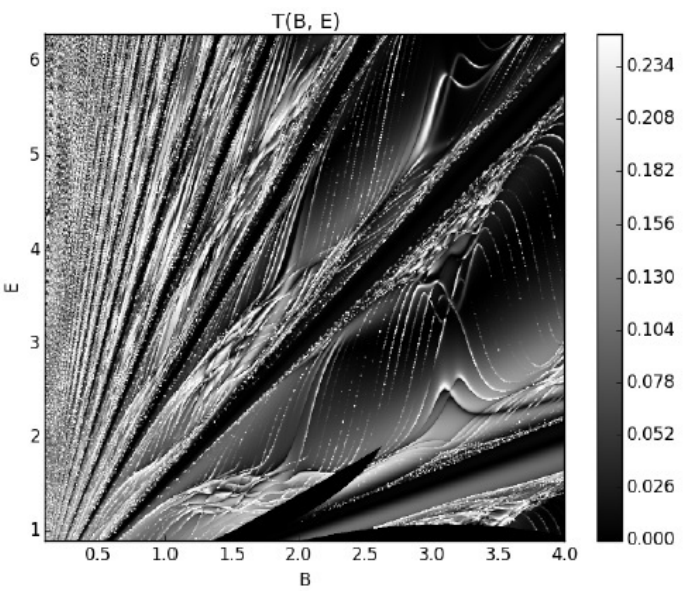

FIG. 5. Dependence of transmission coefficient value $T$ on electron energy $E$ and magnetic field $B$ : case of double-layer square lattice. $B$ and $E$ are in dimensionless units 


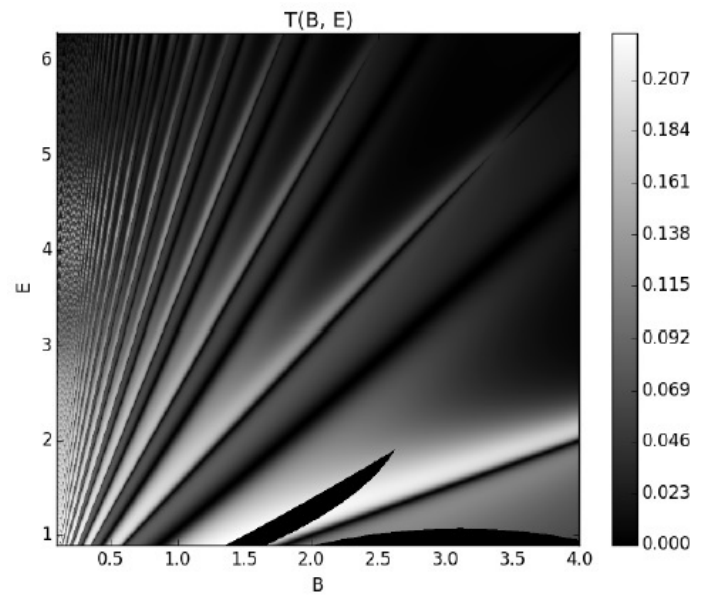

FIG. 6. Dependence of transmission coefficient value $T$ on electron energy $E$ and magnetic field $B$ : case of single-layer hexagonal lattice. $B$ and $E$ are in dimensionless units

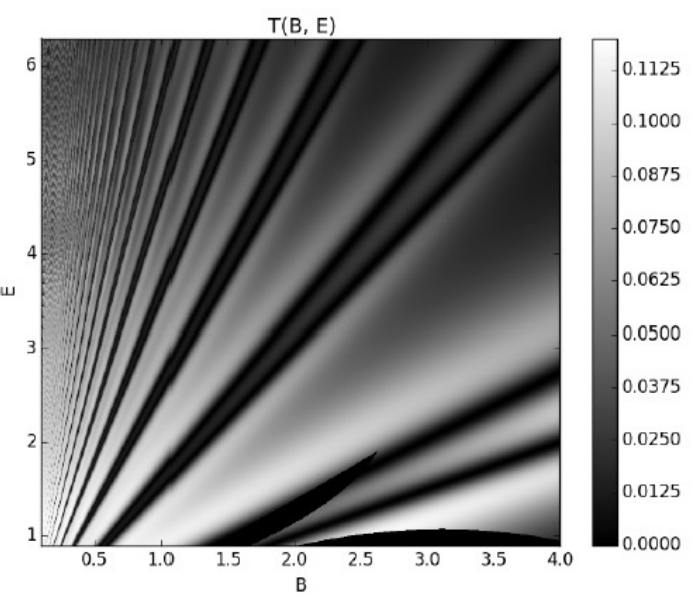

FIG. 7. Dependence of transmission coefficient value $T$ on electron energy $E$ and magnetic field $B$ : case of double-layer hexagonal lattice. $B$ and $E$ are in dimensionless units

It can be observed that the middle of each dark stripe in Fig. 4 and Fig. 5 coincides with some singularity of $\Gamma\left(\frac{1}{2}-\frac{E}{\omega}\right)$ (which is one of multipliers in (3)). The dependence of $T$ on $B$ and $E$ shown in the figures is very complicated. For the hexagonal (honeycomb) lattice, it is also complicated but local oscillations of $T$ have less amplitude, that is why in Figs. 6, 7 looks more monotone. Such effect is related to the complex structure of the spectrum of the Hamiltonian for periodic array of quantum dots. Particularly, it is known that if the number of the magnetic flux quanta through the basic cell of the lattice is rational irreducible fraction $N / M$, then each Landau level (energy level for single center in a magnetic field) splits into $M$ sublevels. For the case of irrational flux, the spectrum has fractal structure. Correspondingly, one has so-called Hofstadter-type "energy-flux" diagram. It was proved theoretically for different cases (see, e.g., [29,30,32]) and was observed experimentally (see, e.g., [34,35]). As for the comparison of tunneling for single-layer and double-layer cases, one observes that in the model with double layer, dark stripes are wider and inside zones between these stripes, $T$ value is, generally, greater. In the case of the hexagonal lattice, when considering double-layer model, dark stripes pairs are closer to each other and value of $T$ in zones between them is less than in the single-layer case. The behavior of $T$ in a neighborhoods of "zero transmission stripes" is shown in details in Figs. 8,9 for a particular value of the magnetic field.

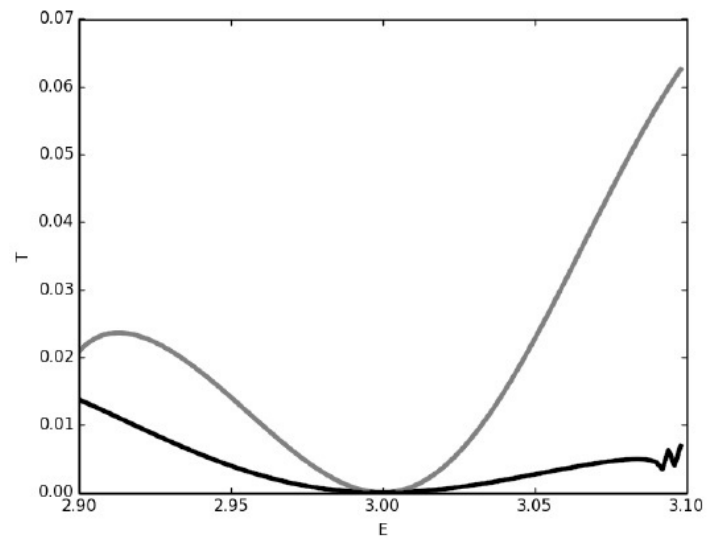

FIG. 8. Comparison of $T(E)$ for $B=2.0$ (square lattice). Black line is for double layer model, grey one is for single layer model. $B$ and $E$ are in dimensionless units

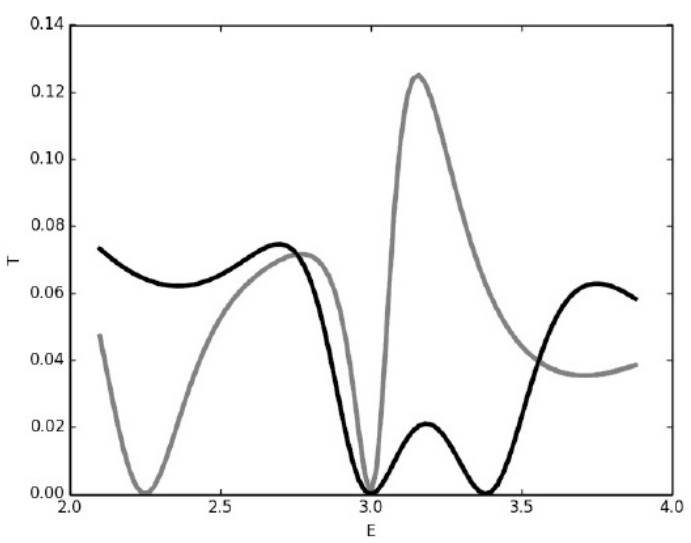

FIG. 9. Comparison of $T(E)$ for $B=2.0$ (hexagonal lattice). Black line is for double layer model, grey one is for single layer model. $B$ and $E$ are in dimensionless units 
It is also interesting to look for the dependence of transmission coefficient value $T$ on the distance $d$ between the layers in the models with channels between the quantum layers. The function $T(d)$ is oscillating. It can be explained by some resonance effects in the quantum structure due to the additional operator for the connecting segments (see Figs. 10 and 11).

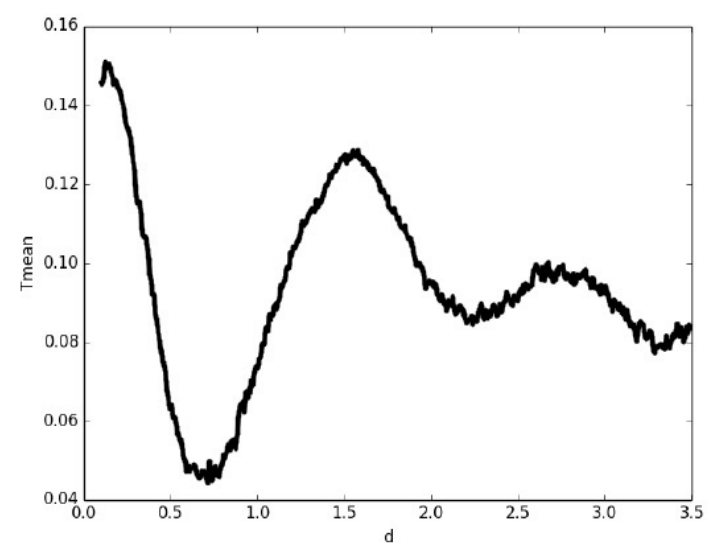

FIG. 10. Dependence of transmission coefficient $T$ on distance between layers $d$ (square lattice). $d$ is in dimensionless units

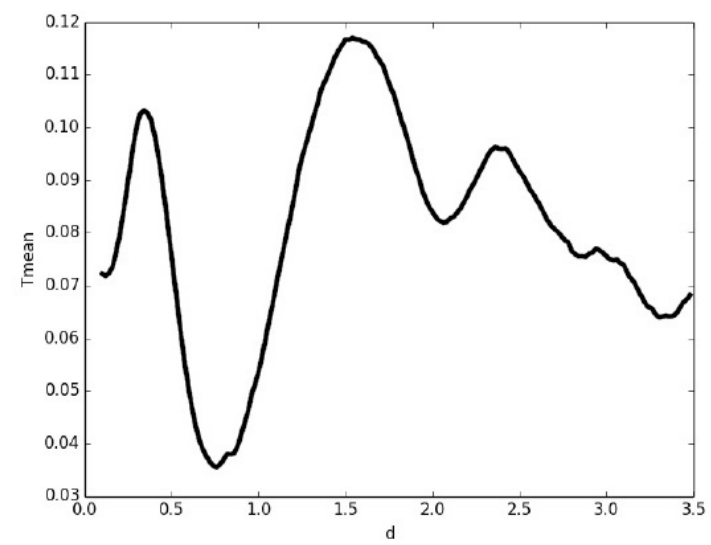

FIG. 11. Dependence of transmission coefficient $T$ on distance between layers $d$ (hexagonal lattice). $d$ is in dimensionless units

\section{Acknowledgements}

This work was partially financially supported by the Government of the Russian Federation (grant 074-U01), by grant MK-5161.2016.1 of the President of the Russian Federation, DFG Grant NE 1439/3-1, by grant 16-11-10330 of Russian Science Foundation.

\section{References}

[1] Hofstadter D.R. Energy levels and wavefunctions of Bloch electrons in rational and irrational magnetic fields. Phys. Rev., 1976, B14, P. 2239-2249.

[2] Gonzalez A., Capote R. Vertical magneto-tunneling through a quantum dot and the density of states of small electronic systems. Physica E, 2001, 10(4), P. 528-534.

[3] Brunner R., Meisels R., et al. Magneto-transport in open quantum dot arrays at the transition from low to high magnetic fields: regularity and chaos. Int. J. Mod. Phys., 2007, B21, P. 1288-1296.

[4] Roco M.C., Mirkin C.A., Hersam M.C. Nanotechnology Research Directions for Societal Needs in 2020. Retrospective and Outlook. Berlin, Springer, 2010.

[5] Bloch I., Dalibard J., Zwerger W. Many-body physics with ultracold gases. Rev. Mod. Phys., 2008, 80, P. 885-964. DOI: 10.1103/RevModPhys.80.885.

[6] Geyler V.A. The two-dimensional Schrödinger operator with a homogeneous magnetic field and its perturbations by periodic zero-range potentials. St. Petersburg Mathematical Journal, 1992, 3(3), P. 489-532.

[7] Mantile A. A linearized model of quantum transport in the asymptotic regime of quantum wells. Nanosystems: Phys. Chem. Math., 2015, 6, P. 100-112.

[8] Dabrowski L., Grosse H. On nonlocal point interactions in one, two, and three dimensions. J. Math. Phys., 1985, 26, P. $2777-2780$.

[9] Albeverio S., Kurasov P. Singular perturbations of differential operators. Solvable Schrödinger type operators. London Mathematical Society Lecture Notes, 2005, $271 \mathrm{pp}$.

[10] Geyler V.A., Pavlov B.S., Popov I.Yu. One-particle spectral problem for superlattice with a constant magnetic field. Atti Sem. Mat. Fis. Univ. Modena., 1998, 46, P. 79-124.

[11] Pavlov B.S. Extensions theory and explicitly-solvable models. Uspekhi Mat. Nauk, 1987, 42(6), P. 99-131.

[12] Geyler V.A., Pavlov B.S., Popov I.Yu. Spectral properties of a charged particle in antidot array: A limiting case of quantum billiard. $J$. Math. Phys., 1996, 37(10), P. 5171-5194.

[13] Popov I.Y., Kurasov P.A., et al. A distinguished mathematical physicist Boris S. Pavlov. Nanosystems: Phys. Chem. Math., 2016, 7(5), P. 782-788.

[14] Eisenstein J.P., et al. New fractional quantum Hall state in double-layer two-dimensional electron systems. Phys. Rev. Lett., 1992, 68(9), P. 1383-1386.

[15] Geyler V.A., Popov I.Yu. Solvable model of a double quantum electron layer in a magnetic field. Proc. Royal Soc. London, 1998, A. 454, P. 697-705. doi: 10.1098/rspa.1998.0181.

[16] Landauer R. Spatial variation of currents and fields due to localized scatterers in metallic conduction. IBM J. Res. Dev., 1957, 49(24), P. 17177-17184. 
[17] Pavlov B.S., Yafyasov A.M. Resonance scattering across the superlattice barrier and the dimensional quantization. Nanosystems: Phys. Chem. Math., 2016, 7(5), P. 816-834.

[18] Popov I.Yu., Osipov S.A. Model of tunnelling through periodic array of quantum dots in a magnetic field. Chinese Phys., 2012, B21, P. 117306.

[19] Eremin D.A., Grishanov E.N., et al. An explicitly solvable model for tunneling through a quantum dots array in a magnetic field. Chinese J. Phys., 2014, 52(24), P. 1100-1109.

[20] Meynster D., Popov A., Popov I. Model of tunnelling through periodic array of quantum dots. ITM Web of Conferences, 2017, 9, P. 01008/1-5.

[21] Bateman H., Erdelyi A. Higher Transcendental Functions (vol. 1). McGraw-Hill, New York, 1953.

[22] Geyler V.A., Popov I.Yu. The spectrum of a magneto-Bloch electron in a periodic array of quantum dots: explicitly solvable model. $Z$. Phys. B, 1994, 93, P. 437-439.

[23] Geerinckx F., Peeters F.M., Devreese J.T. Effect of the confining potential on the magneto optical spectrum of a quantum dot. J. Appl. Phys., 1990, 68(7), P. 3435-3438.

[24] Albeverio S., Gesztesy F., Hoegh-Krohn R., Holden H. Solvable Models in Quantum Mechanics: Second Edition. AMS Chelsea Publishing, Providence, R.I., 2005.

[25] Geyler V.A., Popov I.Yu. Group-theoretical analysis of lattice Hamiltonian with a magnetic field. Phys. Lett., 1995, A, 201, P. 359-364.

[26] Geyler V.A., Popov I.Yu. Periodic array of quantum dots in a magnetic field: irrational flux, honeycomb lattice. Z. Phys., 1995, B98, P. 473-477.

[27] Geyler V.A., Popov I.Yu. Ballistic transport in nanostructures: explicitly solvable model. Theor. Math. Phys., 1996, 107, P. 427-434.

[28] Geyler V.A., Popov I.Yu. Resonant tunnelling in zero-dimensional systems: Explicitly solvable model. Phys. Lett. A, 1994, 187, P. 410-412.

[29] Geyler V.A., Popov I.Yu., Popov A.V., Ovechkina A.A. Fractal spectrum of periodic quantum system in a magnetic field. Chaos, Solitons, and Fractals, 2000, 11(1-3), P. 281-288.

[30] Nemec N., Cuniberti G. Hofstadter butterflies of bilayer graphene. Phys. Rev. B Rapid Comm., 2007, 75, 201404/1-4 (R).

[31] Popov I.Yu., Grishanov E.N. Spectral properties of multi-layered graphene in a magnetic field. Superlatt. Microstruct, 2015 , 86, P. 68-72.

[32] Popov I.Yu., Grishanov E.N. Electron spectrum for aligned SWNT array in a magnetic field. Superlatt. Microstruct., 2016, 100, P. 12761282.

[33] Grishanov E.N., Popov I.Y. Computer simulation of periodic nanostructures. Nanosystems: Phys. Chem. Math., 2016, 7(5), P. 865-868.

[34] Albrecht C., Smet J.H., et al. Evidence of Hofstadter's fractal energy spectrum in the quantized Hall conductance. Phys. Rev. Lett., 2001, 86, P. 147-150.

[35] Wang Z.F., Liu F., Chou M.Y. Fractal Landau-level spectra in twisted bilayer graphene. Nano Lett., 2012, 12, P. 3833-3838. 